IP Periodica Polytechnica

Transportation Engineering

45(4), pp. 223-229, 2017

https://doi.org/10.3311/PPtr.9586

Creative Commons Attribution (i)

RESEARCH ARTICLE

\section{A Study of Metro Organization Based on Multi-objective Programming and Hybrid Genetic Algorithm}

\author{
Jun Zhang ${ }^{1 *}$, Jiang $\mathrm{Li}^{2}$, Yan $\mathrm{Wu}^{1}$
}

Received 12 June 2016; accepted 08 December 2016

\begin{abstract}
Based on the train routing mode ascertained by suitability analysis, we construct a multi-objective problem to optimize the train routing, marshaling number and train headway from the perspective of general cost and segment load ratio by analyzing relevant characteristics like trip time, trip cost, operation cost, spatial distribution characteristics etc. Then the Singular Value Decomposition method and simulation software RailSys have been adopted to calibrate related parameters for the subsequent calculation. By comparison, the Genetic Algorithm is recommended to get an optimal solution to this multi-objective problem, and we improve the traditional algorithm by modifying the coding type, fitness function and crossover operation to enhance the efficiency and convergence. Finally, an operational mode which both satisfies the technological and passenger conditions has been identified to guarantee travellers' safety, improve operation efficiency, save trip time and decrease cost.
\end{abstract}

\section{Keywords}

urban rail transit, train organization, multi-objective programming, genetic algorithm

\footnotetext{
${ }^{1}$ School of Highway, Chang'an University, Xi' an, China

${ }^{2}$ Jinan Urban Construction Group Co. Ltd, Jinan, China

*Corresponding author, e-mail: 2693019978@qq.com
}

\section{Introduction}

Urban Rail Transit has an obvious significance in facilitating sustainable development and land use due to its inherent characteristics like low energy consumption, high occupancy, safety, punctuality etc. In the meanwhile, the organization of train operation plays an important role in the infrastructure design, timetable arrangement and emergency response, only an efficient organization can lead to an efficient operation, therefore it is indispensible to put forward a reasonable and feasible plan upon in-depth analysis of passenger volume's time-distance distribution characteristics.

Many studies have been conducted to optimize the train organization in urban rail transit. First of all, it's necessary to consider the non-equilibrium factors of passenger volume in time and space when analyzing characteristics of a metro line or network (Assis and Milani, 2004; Castelli et al., 2004), which will contribute to a better passenger organization program and a better train operation schedule (Li et al., 2014). Also the multi-service control and related constraints play an important role in metro operation at a high frequency, which minimize the influence caused by timetable bias and consider the rolling stock allocation as well as the basic capacity (Carrel et al., 2010). Meanwhile, the organization scheme should give consideration to the departure interval and travel time while improving the flexibility and efficiency of train operation (Gonzalez et al., 2010).

In the research of model construction, a non-linear model consisiting of transfer walking time and waiting time which is driven by departure and arrival events has been established, and evolutional algorithm is applied to solve the problem (Wang et al.,2015). Despite passengers' travel time, energy consumption has also been given consideration to optimize the timetable for peak and off-peak hours by genetic algorithms (Xu et al., 2016). Besides, a bi-level planning model composed of operation scheme and passenger selection has been proposed to work out the train interval and marshaling number, oriented by Stacklberg gaming relation under user equilibrium (Deng et al., 2012).

Our study starts with analysis on basic infrastructure data of Xi'an Metro Line 4 to ensure a reasonable train routing, combined with the forecasted passenger volume O-D matrix, 
then focuses on the optimal objectives to search for a systematic and coordinative train operation plan during the peak hour.

\section{Train Routing}

Besides the engineering conditions, the demands of every section's passenger volume should be taken into account when making the train routing mode for the subsequent operation plan. We can confirm a preliminary routing mode from 5 th segment to 21 st segment by the criterion of $1 / 2$ maximum segment passenger volume, as shown in Fig. 1.

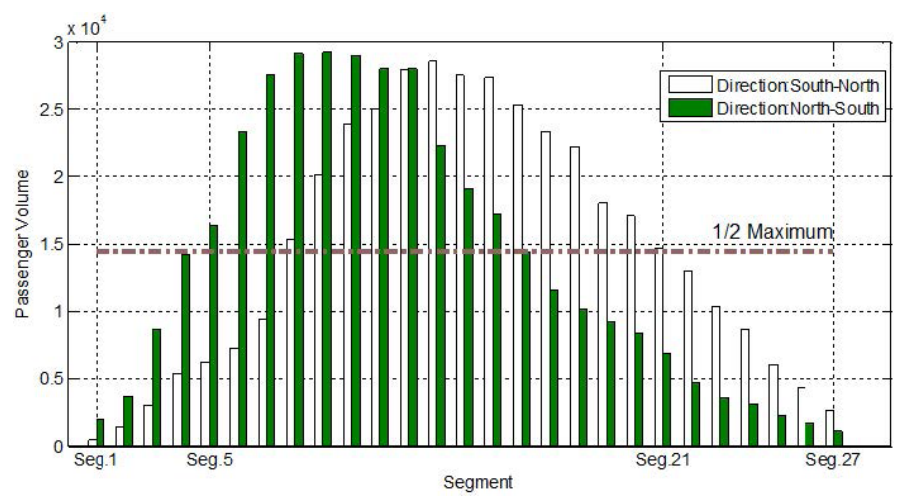

Fig. 1 Line screen passenger volume in peak hour

However, we'd better analyse the suitability of every possible turn-back station under continuous high passenger volume section in order to increase the occupancy and save the rolling stock. The suitability can be calculated as follows.

$$
S_{i}=\frac{\left(1-p_{i 1}\right)+\left(1-p_{i 2}\right)}{2} .
$$

Where $p_{i 1}$ and $p_{i 2}$ are the passenger volume ratio of two adjacent segments at station $i$, on up-direction and downdirection respectively.

From Eq. (1) we can know that the value of suitability represents the change of segment passenger volume, the larger it is, the more reasonable and suitable to consider the station as turn-back station. In this way, the $6^{\text {th }}$ station and $22^{\text {nd }}$ station are better to be turn-back stations, the calculation results are shown in Table 1.

Table 1 Calculation results of each station's suitability

\begin{tabular}{lllllll}
\hline Sta.1 & Sta.2 & Sta.3 & Sta.4 & Sta.5 & Sta.6 & Sta.7 \\
-- & 0.58 & 0.55 & 0.409 & 0.132 & $\mathbf{0 . 2 2 1}$ & 0.192 \\
\hline Sta.8 & Sta.9 & Sta.10 & Sta.11 & Sta.12 & Sta.13 & Sta.14 \\
0.222 & 0.121 & 0.082 & 0.038 & 0.054 & 0.113 & 0.089 \\
\hline Sta.15 & Sta.16 & Sta.17 & Sta.18 & Sta.19 & Sta.20 & Sta.21 \\
0.051 & 0.121 & 0.137 & 0.086 & 0.138 & 0.073 & 0.161 \\
\hline Sta.22 & Sta.23 & Sta.24 & Sta.25 & Sta.26 & Sta.27 & sta.28 \\
$\mathbf{0 . 2 1 7}$ & 0.21 & 0.151 & 0.288 & 0.26 & 0.376 & -- \\
\hline
\end{tabular}

However the $22^{\text {nd }}$ station will be the transfer station connecting metro line 4 and line 2 , to reduce the difficulty and complexity of operation management and ensure an ordered transfer process, we finally ascertain the $6^{\text {th }}$ station and $21^{\text {st }}$ station to be the turn-back stations of short routing combined with the preliminary train routing organization, as shown in Fig. 2.

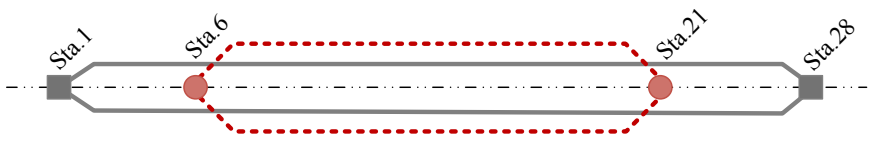

Fig. 2 General view of train routing mode

\section{Objective Functions}

Before constructing objective functions, the following hypotheses are in need:

(1) Passenger from station at short routing to station at long routing always choose long routing trains regardless of transfer;

(2) Under the circumstance of high density operation, average waiting time is half of the train departure interval;

(3) Trains stop at every stop, the dwell time is classified into 3 types according to the volume scale, specific values are 20s, 40s and 60s in turns;

(4) Pairs of trains running on short routing should be an integral multiple of trains on long routing to avoid unoccupied time period;

(5) A-type locomotives are applied, and the rated passenger capacity $\rho$ is about 310 people per carriage under standard density 6 people $/ \mathrm{m}^{2}$.

Explanation of symbols:

Station denoted by $s$, passenger volume from station $s$ to station $i$ denoted by $a_{s i}$, up-direction and down-direction denoted by $u$ and $d$, original and terminal stations of short routing are $p$ and $q, \mathrm{~m}$ represents total number of stations, $D$ represents dwell time at different station (varies in station type).

$\alpha$ and $\beta$ stand for the marshaling number of long routing and short routing respectively. $r$ is the ratio of long routing trains to short routing trains, if the interval of long routing is $I_{1}$, the interval of short routing is $I_{2}$, then $I_{2}=I_{1} /(1+r)$.

To enhance the accuracy and applicability, we establish the objective functions from perspectives of generalized cost and segment load ratio.

\subsection{Generalized Cost}

Generalized cost can be expressed by adding weightsum of passengers' time cost, passengers' monetary cost and system operation cost. The first objective function $Z_{1}$ can be constructed as follows

$$
\min Z_{1}=\omega_{1} T \cdot V O T+\omega_{2} M+\omega_{3} F .
$$


Where $\omega_{1}, \omega_{2}$ and $\omega_{3}$ are the weighting coefficients of time cost, monetary cost and operation cost respectively; VOT is the value of time, the average level is about 0.35 Yuan/min per trip according to random sampling survey on commuting passengers.

\section{Time Cost}

Passengers' travel time is the time spent on metro transit, exclusive of the waiting time, which can be constructed as

$$
T_{\text {travel }}=\sum_{i=1}^{m-1} n_{i}^{u} \cdot \frac{l_{i, i+1}}{v_{i, i+1}^{u}}+\sum_{i=1}^{m-1} n_{i}^{d} \cdot \frac{l_{i, i+1}}{v_{i, i+1}^{d}} .
$$

Where $l_{i, i+1}$ is the length of $i^{\text {th }}$ segment, $v_{i, i+1}^{u}$ and $v_{i, i+1}^{d}$ are the average velocity of up-direction and down-direction in $i^{\text {th }}$ segment, $n_{i}^{u}$ and $n_{i}^{d}$ are the passenger volume of up-direction and down-direction in $i^{\text {th }}$ segment.

Waiting time concludes the passengers' average waiting time and trains' dwell time, defined in the following norms different in position and direction.

If the station s only belongs to long routing, namely when $s \in\{[1, p-1] \cup[q+1, m]\}$, we can get

$$
\begin{gathered}
T_{\text {wait }}^{u 1}=\sum_{s=1}^{p-1} \sum_{i=s+1}^{m} a_{s i} \cdot\left(\frac{I_{1}}{2}+D_{s}\right)+\sum_{s=q+1}^{m} \sum_{i=s+1}^{m} a_{s i} \cdot\left(\frac{I_{1}}{2}+D_{s}\right), \\
T_{\text {wait }}^{d 1}=\sum_{s=1}^{p-1} \sum_{i=1}^{s-1} a_{s i} \cdot\left(\frac{I_{1}}{2}+D_{s}\right)+\sum_{s=q+1}^{m} \sum_{i=1}^{s-1} a_{s i} \cdot\left(\frac{I_{1}}{2}+D_{s}\right) .
\end{gathered}
$$

Where $T_{\text {wait }}^{u 1}$ and $T_{\text {wait }}^{d 1}$ are the total waiting time of station belong to the long routing on up-direction and down-direction respectively.

If station $s$ belongs to both short routing and long routing, namely when $s \in[p, q]$, we can get

$$
\begin{aligned}
& T_{\text {wait }}^{u 2}= \\
& \sum_{s=p}^{q}\left[\left(\sum_{i=s+1}^{m} a_{s i}-\sum_{i=q+1}^{m} a_{s i}\right) \cdot\left(\frac{1}{2} \cdot \frac{I_{1}}{1+r}+D_{s}\right)+\sum_{i=q+1}^{m} a_{s i} \cdot\left(\frac{I_{1}}{2}+D_{s}\right)\right],
\end{aligned}
$$

$$
\begin{aligned}
& T_{\text {wait }}^{d 2}= \\
& \sum_{s=p}^{q}\left[\left(\sum_{i=1}^{s-1} a_{s i}-\sum_{i=1}^{p-1} a_{s i}\right) \cdot\left(\frac{1}{2} \cdot \frac{I_{1}}{1+r}+D_{s}\right)+\sum_{i=1}^{p-1} a_{s i} \cdot\left(\frac{I_{1}}{2}+D_{s}\right)\right] .
\end{aligned}
$$

Where $T_{\text {wait }}^{u 2}$ and $T_{\text {wait }}^{d 2}$ are the total waiting time of up-direction and down-direction at stations belong to the short routing respectively.

According to Eqs. (4) to (7), total time cost $T_{c}$ can be obtained as

$$
T_{c}=\left(T_{\text {travel }}+T_{\text {wait }}^{u 1}+T_{\text {wait }}^{u 2}+T_{\text {wait }}^{d 1}+T_{\text {wait }}^{d 2}\right) \cdot V O T \text {. }
$$

\section{Monetary Cost}

Passengers' monetary cost can be calculated as

$$
M=\sum_{s=1}^{m} \sum_{i=1}^{m} a_{s i} \cdot l_{s, i} \cdot c .
$$

Where $l_{s, i}$ is the distance between station $s$ and station $i, c$ is unit cost per kilometre, here $c$ equals about 0.2 Yuan $/ \mathrm{km}$ in Xi'an Metro.

\section{Operation Cost}

During peak hour, taken the circulation length and time of each routing mode into consideration, the train operation cost can be calculated as

$$
F=\left(\alpha \cdot \frac{60}{I_{1}} \cdot 2 \sum_{i=1}^{m-1} l_{i, i+1}+\beta \cdot r \frac{60}{I_{1}} \cdot 2 \sum_{i=p}^{q-1} l_{i, i+1}\right) \cdot g .
$$

Where $g$ represents unit carriage cost per kilometer,Yuan $/ \mathrm{km}$.

\subsection{Segment Load Ratio}

In order to ensure non-negativity, the DQS algorithm (Difference Quadratic Sum) has been adopted to compare the segment ratio of volume to line capacity, the higher the ratio is, the smaller the DQS will be. DQS are constructed as

$$
\begin{aligned}
Z^{u} & =\sum_{\substack{i=1 \\
i \notin[p, q]}}^{m-1}\left(\frac{n_{i}^{u}}{\frac{\alpha \rho \cdot l}{m-1}}-1\right)^{2}+\sum_{i=p}^{q}\left(\frac{n_{i}^{u}}{\frac{\beta \rho \cdot r l}{q-p}+\frac{\alpha \rho \cdot l}{m-1}}-1\right)^{2}, \\
Z^{d} & =\sum_{\substack{i=1 \\
i \notin[p, q]}}^{m-1}\left(\frac{n_{i}^{d}}{\frac{\alpha \rho \cdot l}{m-1}}-1\right)^{2}+\sum_{i=p}^{q}\left(\frac{n_{i}^{d}}{\frac{\beta \rho \cdot r l}{q-p}+\frac{\alpha \rho \cdot l}{m-1}}-1\right)^{2} .
\end{aligned}
$$

Where $Z^{u}$ and $Z^{d}$ are the DQS of entire line's segment load ratio on up-direction and down-direction.

Combining Eqs. (11) and (12), the second objective function $Z_{2}$ can be expressed in the following norm

$$
\min Z_{2}=\left(Z^{u}+Z^{d}\right) /(2 m-2) \text {. }
$$

\section{Calibrating Multi-objective Functions 4.1 Weight Calculation}

Based on the survey and consultation to passengers and operation managers about factors affecting service and operation, we used Rough Set theory (Liang et al., 2013) to construct deterministic matrix by importance transform for decision-making factors' weight calculation, which conclude trip time, trip cost, operation cost, spatial characteristics and degree of comfort. The relation between every factor's Linguistic Value (LV) and Triangular Fuzzy Number (TFN) is shown in Table 2. 
Table 2 Correspondence between LV and TFV

\begin{tabular}{llll}
\hline $\mathbf{L V}$ & TFN $(\boldsymbol{A})$ & $\mathbf{L V}$ & TFN $(\boldsymbol{A})$ \\
\hline Very Bad & $(0,0,20)$ & Better & $(60,70,80)$ \\
Bad & $(0,10,30)$ & Good & $(70,80,90)$ \\
Poor & $(0,20,40)$ & Very Good & $(80,90,100)$ \\
Normal & $(30,50,70)$ & Superlative & $(90,100,100)$ \\
\hline
\end{tabular}

Let's write the TFN as $A$, namely $A=(f, g, h)$, then the quantitative evaluation result of $A$ can be obtained by the equation below

$$
D(A)=\frac{1}{5}(f+g+g+g+h)=\frac{1}{5}(f+3 g+h) .
$$

After normalizing the quantified deterministic matrix, it's a conformity acceptable matrix upon conformity test. Therefore the SVD (Singular Value Decomposition) method can be adopted to calculate each factor's weight (Gass and Rapcsák, 2004). The calculated weights of trip time, trip cost, operation cost and spatial characteristics are $0.176,0.059,0.173$ and 0.317 respectively.

\subsection{Multi-objective Functions}

Based on the future estimated OD matrix of $4^{\text {th }} \mathrm{Xi}$ 'an Metro Line, combined the train routing mode demonstrated in paragraph 2 with the diagram exported by the simulation system RailSys, according to Eqs. (3) to (10), we can get

$$
\begin{gathered}
T_{\text {wait }}=23216.5 I_{1}+33476 I_{1} /(1+r)+64368 . \\
M=907004.33 \cdot c . \\
F=\left(3957.12 \alpha / I_{1}+1926.48 r \beta / I_{1}\right) \cdot g .
\end{gathered}
$$

Partial topological graphs built by simulation software RailSys are shown in Fig. 3.

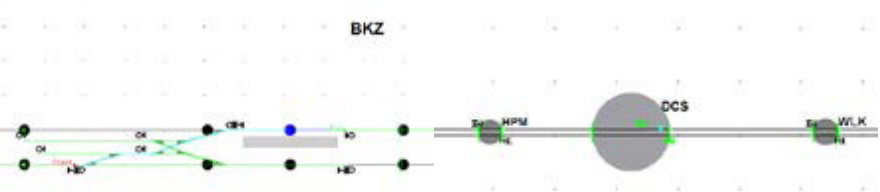

Fig. 3 Station topology and segmenttopology

According to factors' weights, when plugging Eqs. (15) to (17) into Eq. (2), we can obtain the first objective $Z_{1}$ as

$$
\begin{aligned}
\min Z_{1}= & 1483.12 I_{1}+2056.26 I_{1} /(1+r) \\
& +684581.76 \alpha / I_{1}+333281.04 r \beta / I_{1}+14565.76 .
\end{aligned}
$$

Furthermore, based on the passenger volume distribution in peak hour and the standard passenger capacity per train, the second objective $Z_{2}$ is obtained as

$$
\begin{aligned}
\min Z_{2}= & 53.57 \frac{I_{1}^{2}}{\alpha^{2}}-7.78 \frac{I_{1}}{\alpha}+7.01 /\left(\frac{r \beta}{5 I_{1}}+\frac{\alpha}{9 I_{1}}\right)^{2} \\
& -3.73 /\left(\frac{1}{5} \cdot \frac{r \beta}{I_{1}}+\frac{1}{9} \cdot \frac{\alpha}{I_{1}}\right)+1
\end{aligned}
$$

\subsection{Restrictions}

The train interval should both satisfy the passengers' waiting time and the demands of signal control system. In Xi'an, the average patient waiting time is about 6 minutes and the extreme interval limit is 1.5 minutes, namely $1.5 \leq I_{1} \leq 6$ and $1.5 \leq I_{1}(1+r) \leq 6$, hence we can get $1.5(1+r) \leq I_{1} \leq 6$.

The interval for short routing should be an integral multiple of interval for long routing in order to keep a balance of interval in each segment. Taking the complexity of operation and device conditions into account, the ratio $r$ should better be 1 or 2 , namely we consider $r \in\{1,2\}$.

The maximum marshaling of a train consists of 8 carriages on the ground of the design form of platform of Line 4.8 marshaling carriages for the long routing trains are necessary to provide a basic transit capacity for the whole line, and the marshalling for the short routing trains should be at least 5 for the crowded segments, namely $\alpha=8$ and $\beta \in\{5,6,7,8\}$.

\section{Advanced Hybrid Genetic Algorithm 5.1 Genetic Algorithm Overview}

Classic algorithms used for optimization problems are Hillclimbing method, Simulated Annealing method, Exhaustion method, Heuristic method, etc. Genetic Algorithm has the following advantages over classic algorithm:

(1) Searching points in colony are parallel, and the searching procedure based on surface is better than traditional search based on single point unit;

(2) No auxiliary information is in need, the objective function and relevant fitness which affect the searching direction are enough;

(3) GA emphasizes using probability transformation rules not the certainty rules to guide the searching procedure;

(4) The parameter set is established by digitized code, which reflects the relationship between phenotype and genotype.

Compared with typical GA, we conduct the algorithm by some modifications and thus constitute the hybrid genetic algorithm (HGA). Firstly, we use the Gray code to digitize the variables which can enhance the local searching ability and make the crossover and mutation operation easier to realize when compared to Binary code. Secondly, the fitness function is designed by Boundary Construction method based on objective functions, which can guarantee the probability's non-negativity and prevent a larger disparity in the distribution of objective values, as well as embody the colony's average performance by 
calculating the average fitness. Thirdly, two-point crossover is adopted to improve the computational efficiency on the basis of excellent genotype's continuity. Concrete steps are as follows:

Step1. (Coding) Use Gray code to digitize the variable in solution space to chromosome in genetic space.

Step2. (Initialization) Generate original colony and set the maximum count of generations' iteration. Here the initial colony size is 40 and the max of generation quantity is 200 , the generation gap is set as 0.95 .

Step3. (Selection) Select by individual's fitness value, individuals with higher fitness value have a higher probability to be inherited in filial generation, so that the colony's average fitness can continuously approach the optimal solution, the fitness function is constructed in the following equation

$$
\text { FitV }(P o s)=2-s p+2 \times(s p-1) \times \frac{P o s-1}{\text { Nind }} .
$$

Step4. (Recombination) According to the two selected individuals with a higher probability among the colony, set two random crossover points in the digitized chromosome before exchanging part of genes to produce progenies, the crossover probability is set as 0.7 .

Step5. (Mutation) By changing position values of individual chromosomes in a small probability to produce new individuals, so that can avoid information loss caused by selection and crossover operation and improve algorithm's effectiveness, the mutation probability is 0.001 .

\subsection{Solution of Compound Objective Problem}

To solve the multi-objective functions, we adopt compound objective function by introducing the degree of scaling to erase dimensionality of objectives $Z_{1}$ and $Z_{2}$. The hybrid objective function can be established in the following equation by jointing two objectives according to the factors' weights in 4.1.

$$
\begin{aligned}
& \min Z=\eta_{1} \cdot \frac{Z_{1}-Z_{1 \min }}{\left(Z_{1 \max }-Z_{1 \min }\right)}+\eta_{2} \cdot \frac{Z_{2}-Z_{2 \min }}{\left(Z_{2 \max }-Z_{2 \min }\right)} \\
& \text { s.t. }\left\{\begin{array}{c}
r \in\{1,2\} \\
1.5(1+r) \leq I_{1} \leq 6 \\
\alpha=8, \quad \beta \in\{5,6,7,8\}
\end{array} .\right.
\end{aligned}
$$

Where $\eta_{1}$ and $\eta_{2}$ are the weights of objective $Z_{1}$ and $Z_{2}$ respectively, the former is the aggregate weight of passenger cost and system operation cost, the latter is the weight of spatial distribution characteristics.

(1) When $r=1$, according to Eqs. (18) and (19), related parameters can be calculated as follows.

For objective $\mathrm{Z}_{1}$ :

We have $Z_{1 \max }=2.7364 \times 10^{6}$ and $Z_{1 \min }=1.2201 \times 10^{6}$, hence the scaling factor $p_{1}=1.5163 \times 10^{6}$.

For objective $\mathrm{Z}_{2}$ :
We have $Z_{2 \max }=84.1805$ and $Z_{2 \min }=11.3405$, hence the scaling factor $p_{2}=72.876$.

Combined with Eq. (20), we can get $I_{1}=5.2529$ and $\beta=8$, the minimum value of compound objective function $\mathrm{Z}$ is 0.3191, as shown in Fig. 4.

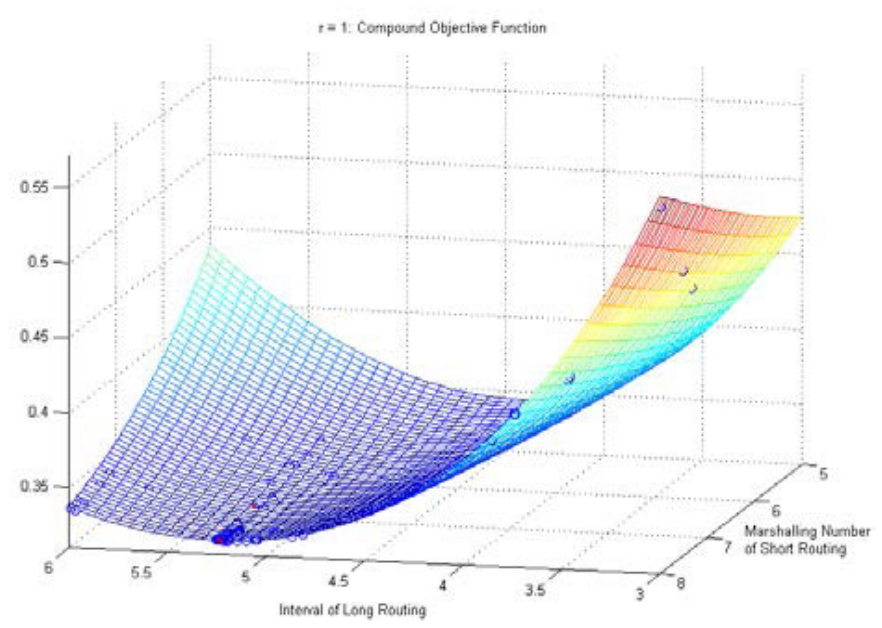

Fig. 4 Objective function' surface diagram and searching points $(r=1)$

The GA algorithm starts to converge through approximate 20 iterations of generation, as shown in Fig. 5.

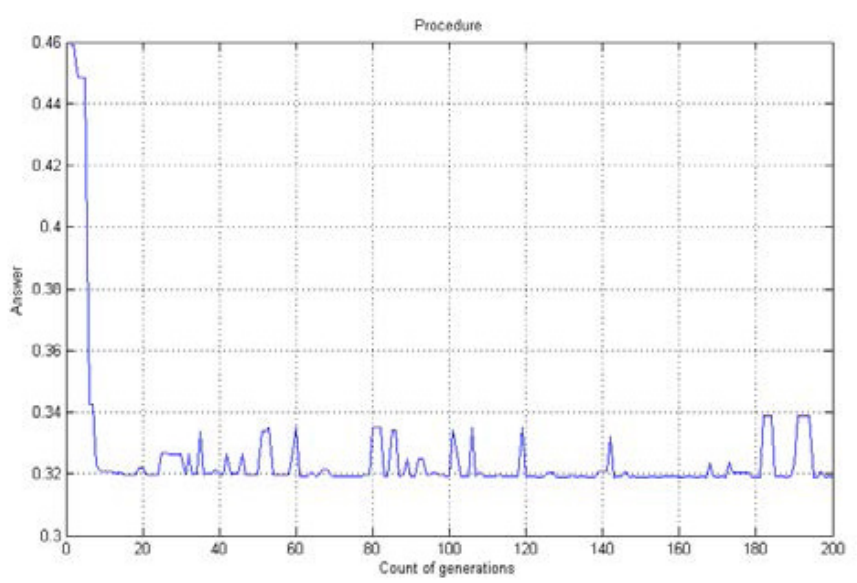

Fig. 5 Objective function value's convergence with generations $(r=1)$

(2) When $r=2$, according to Eqs. (18) and (19), related parameters can be calculated as follows

For objective $Z_{1}$ :

We have $Z_{1 \max }=2.4264 \times 10^{6}$ and $Z_{1 \min }=1.4958 \times 10^{6}$, hence the scaling factor $p_{1}=0.9306 \times 10^{6}$.

For objective $Z_{2}$ :

We have $Z_{2 \max }=47.7896$ and $Z_{2 \min }=17.9591$, hence the scaling factor $p_{2}=29.8305$.

Combined with Eq. (20), we can get $I_{1}=5.0625$ and $\beta=5$, the minimum value of compound objective function $\mathrm{Z}$ is 0.3767, as shown in Fig. 6.

The GA algorithm starts to converge through approximate 70 iterations of generation, shown in Fig. 7. 


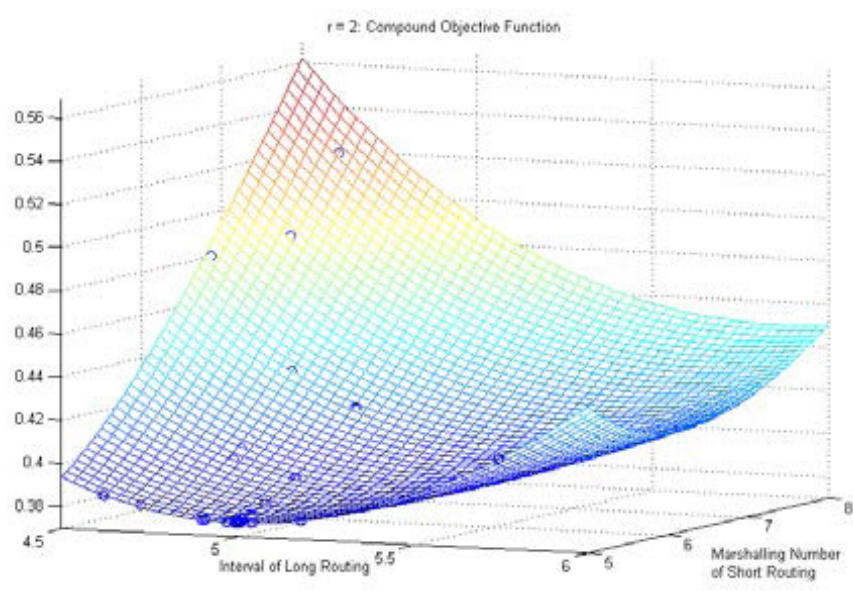

Fig. 6 Objective function' surface diagram and searching points $(r=2)$

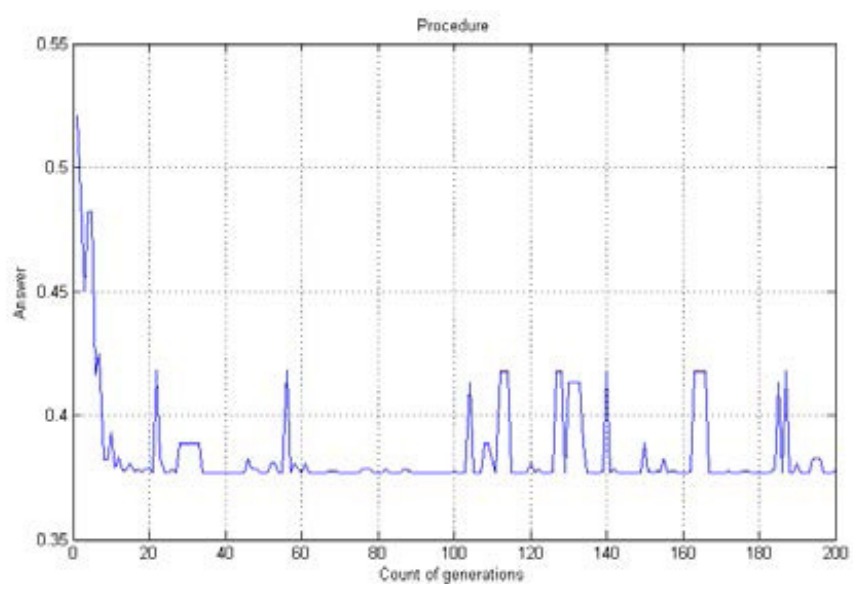

Fig. 7 Objective function value's convergence with generations $(r=2)$

\subsection{Comparison and Selection}

From the above calculation, we can identify two initial train organization schemes for Line 4 in peak hours as follows.

$1^{\text {st }}$ scheme: Interval $I_{1}$ for long routing is $5 \mathrm{~min} 16 \mathrm{~s}$, relevant marshaling number $\alpha$ is 8 . Interval $I_{2}$ for short routing is $2 \mathrm{~min} 38 \mathrm{~s}$, relevant marshaling number $\beta$ is 8 . Ratio $r$ of pairs of trains in long routing to short routing is 1 .

$2^{\text {nd }}$ scheme: Interval $I_{1}$ for long routing is $5 \mathrm{~min} 4 \mathrm{~s}$, relevant marshaling number $\alpha$ is 8 . Interval $I_{2}$ for short routing is $1 \mathrm{~min} 41 \mathrm{~s}$, relevant marshaling number $\beta$ is 5 . Ratio $r$ of pairs of trains in long routing to short routing is 2 .

In order to finally reach a better organization, two schemes are compared with each other from the perspective of theory and practice.

\section{Theoretically Comparison}

Compound objective function value for $1^{\text {st }}$ scheme is 0.319 , where objective $Z_{1}=2.0837 \times 10^{6}$ and objective $Z_{2}=42.3406$;

Compound objective function value for $2^{\text {nd }}$ scheme is 0.377 , where objective $Z_{1}=2.1872 \times 10^{6}$ and objective $Z_{2}=37.8609$.
Obviously, the first scheme has a lower general cost, but its segment load factors DQS is a little high when compared with the second scheme.

\section{Practically Comparison}

Since the departure interval of short routing $I_{2}$ in $2^{\text {nd }}$ scheme is $1 \mathrm{~min} 41 \mathrm{~s}$, which is extremely close to the limit of $1 \mathrm{~min} 30 \mathrm{~s}$ under the signal control system and have a rigorous demand for turn-back infrastructure conditions. Meanwhile, the marshaling number of short routing $\beta$ in $2^{\text {nd }}$ scheme is 5 , which will bring about difficulties in organizing platform passengers.

Above all, the $1^{\text {st }}$ scheme is more reasonable and feasible for the future train organization of Line 4 during peak hours.

\section{Conclusion}

In this study about the train operation organization in Urban Rail Transit, decision factors like passengers' trip cost, operation cost and spatial characteristics are taken into account to establish the structure of multi-objective problem. Meanwhile, SDV algorithm and RailSys simulation are indispensible to calibrate the parameters during modeling procedure. To get an optimal solution of the multi-objective problem, we use hybrid genetic algorithm by improving the classic algorithm so that the algorithm's convergence and result's stability get enhanced to some degree. The final train operational scheme can accommodate to the real train performance, which demonstrates the feasibility and reliability of this multi-objective model.

However, there is a need for continued or more in-depth discussion in certain topics because this study focus on a singular metro line irrespective of the impact of other lines in whole city's metro network, and some hypotheses have been made before constructing the multi-objective model. Meanwhile, when using simulation software to calibrate parameters like velocity and time, errors are inevitable due to the difference between fixed block system and CBTC system.

\section{Acknowledgement}

The project presented in this article is supported by the Key Programs of Department of Transport of Shaanxi, China (No.14-29R). The authors gratefully acknowledge Xi'an Metro Corporation for data collection efforts.

\section{References}

Assis, W. O., Milani, B. E. A. (2004). Generation of optimal schedules for metro lines using model predictive control. Automatica. 40(8), pp. 1397-1404. https://doi.org/10.1016/j.automatica.2004.02.021

Carrel, A., Mishalani, R., Wilson, N. Attanucci, J. Rahbee, A. (2010). Decision Factors in Service Control on High-Frequency Metro Line Importance in Service Delivery. Transportation Research Record: Journal of the Transportation Research Board. 2146(7), pp. 52-59.

https://doi.org/10.3141/2146-07 
Castelli, L., Pesenti, R., Ukovich, W. (2004). Scheduling multimodal transportation systems. European Journal of Operational Research. 155(3), pp. 603-615. https://doi.org/10.1016/j.ejor.2003.02.002

Deng, L. B., Zeng, Q., Gao, W., Zhou, W. L. (2012) Research on train plan of urban rail transit with elastic demand. Journal of the China Railway Society. 34(12), pp. 16-25.

https://doi.org/10.3969/j.issn.1001-8360.2012.12.003

Gass, S. I., Rapcsák, T. (2004). Singular value decomposition in AHP. European Journal of Operational Research. 154(3), pp. 573-584. https://doi.org/10.1016/S0377-2217(02)00755-5

Gonzalez, J., Rodriguez, C., Blanquer, J., Mera, J. M., Castellote, E., Santos, R. (2010). Increase of metro line capacity by optimization of track circuit length and location: In a distance to go system. Journal of Advanced Transportation. 44(2), pp. 53-71.

https://doi.org/10.1002/atr.109

Li, W. Y., Wang, Z. Y. R., Qing, Y., Yu, H. F. (2014). Analysis the characteristics of Beijing Metro Line 4 passenger flow. In: ICIA 2014. Hulunbuir, China. Jul. 28-30, 2014, pp. 76-80.

https://doi.org/10.1109/ICInfA.2014.6932629
Liang, D. C., Liu, D., Pedrycz, W., Hu P. (2013). Triangular fuzzy decisiontheoretic rough sets. International Journal of Approximate Reasoning. 54(8), pp. 1087-1106. https://doi.org/10.1016/j.ijar.2013.03.014

Wang, Y. H., Tang, T., Ning, B., Ton, J. J., Bart, D. D. (2015) Passenger-demands-oriented train scheduling for an urban rail transit network. Transportation Research Part C: Emerging Technologies. 60(11), pp. 1-23. https://doi.org/10.1016/j.trc.2015.07.012

Xu, X. M., Li, K. P., Li, X. (2016) A multi-objective subway timetable optimization approach with minimum passenger time and energy consumption. Journal of Advanced Transportation. 50(1), pp. 69-95. https://doi.org/10.1002/atr.1317 